\title{
GPAS - Graphical Password Authentication System for Software Privacy Protection
}

\author{
S.Geetha, N.Thilagavathi, A.Nivedha shree, M.Subalakshmi
}

\begin{abstract}
Now daily's larger part of PC frameworks, passwords is the technique for decision for validating clients. A procedure by which a framework confirms the personality of a client is known as 'Confirmation'. Confirmation may likewise be summed up by saying that "to validate" signifies "to approve". Confirmation is the main line of resistance against trading off classification and uprightness. The most generally and normally utilized confirmation is conventional "Username" and "Secret phrase". For such verification by and large content (alphanumeric) is utilized. It is outstanding, in any case, that passwords are defenseless to assault: clients will in general pick passwords that are anything but difficult to recollect, and regularly this implies they are likewise simple for an assailant to get via hunting down hopeful passwords. Token and biometric based validation frameworks were presented as an option for those plans. Be that as it may, these plans are all around expensive. Consequently, Graphical plan was acquainted as a variety with the login/secret key plan. In this paper we investigate a way to deal with client confirmation that sums up the idea of a literary secret key and that, as a rule, improves the security of client verification over that given by printed passwords. In this proposed framework we have utilized another system for confirmation. It is a variety to the login/secret phrase plan utilizing graphical secret phrase utilized in a graphical way. We have presented a structure of our proposed Graphical Password Authentication System (GPAS), which is resistant to the basic assaults endured by other confirmation plans.
\end{abstract}

Keywords:Computer Systems, Authentication, Alpha-Numeric, Password Attack, Graphical Password Authentication System (GPAS).

\section{INTRODUCTION}

Verification is a procedure of deciding if a specific individual or a gadget ought to be permitted to get to a framework or an application or essentially an item running in a gadget. Verification procedure guarantees the essential security objectives, for example classification and honesty. The principal line of safeguard for securing any asset is Authentication. For secure validation it is important that a similar verification procedure may not be utilized in each situation. For instance, getting to a "talk server" a less validation security strategy is utilized when contrasted with getting to a corporate database. The agreeableness of any confirmation conspire extraordinarily relies upon its strength against assaults just as its asset necessity both at the customer and at the server end. Because of the multiplication of portable and hand-held gadgets the asset

Revised Manuscript Received on April 12, 2019.

S.Geetha,Department of computer science and engineering, V.S.B Engineering College, Karur, Tamil Nadu, India (Email: geetha1995s@gmail.com)

N.Thilagavathi,Department of computer science and engineering, V.S.B Engineering College, Karur, Tamil Nadu, India

A.Nivedha shree,Department of computer science and engineering, V.S.B Engineering College, Karur, Tamil Nadu, India

M.Subalakshmi,Department of computer science and engineering, V.S.B Engineering College, Karur, Tamil Nadu, India necessity has turned into a main consideration. Presently multi day's clients can get to any data including banking and corporate database, by utilizing cell phones. For banking we propose our Authentication System utilizing Graphical Password, in which the plan enables any picture to be utilized and it doesn't require fake predefined click locales with well-checked limits. Graphical Password can be framed in the mix of Image Icons or Pictures. As such, graphical secret key is a validation framework that works by having the client select from pictures, in a particular request, introduced in a graphical UI (GUI). Consequently, the graphical-secret phrase approach is some of the time called graphical secret key verification framework (GPAS). In GPAS, the server has secret word at the season of validation and at the season of enlistment, the client gives this data to the server in a graphical structure at the season of enrolment and login. PC and Information security is especially subject to secret phrase for the confirmation of the clients and are normal by and by. There are a few confirmation plans accessible in the writing. They can be comprehensively named: Knowledge based validation, Token based confirmation and Biometric based verification. A case of the "Learning based validation" is the customary username/secret phrase or PIN based confirmation plot. Instances of "Token based validation" are Smartcards or electronic tokens end at last biometric based verification plans are instances of the "Biometric based confirmation" kind of validation. Some confirmation frameworks may utilize a blend of the above plans. In GPAS, we centre just around "Information based verification" kinds of confirmation.

\section{LITERATURE REVIEW}

Content technique is most generally utilized, since it is anything but difficult to execute and utilize. One of the fundamental entanglements in content based secret word is the trouble of recalling that it. There were part of issues in utilizing conventional alphanumeric secret key, for example, defenseless against speculating, lexicon assault, keylumberjack, overlook secret key, bear surfing and social building, in spite of the fact that they are generally utilized. Studies have demonstrated that clients will in general pick short and simple passwords that can be utilized by them effectively. Be that as it may, these passwords can likewise be effectively speculated or broken. Content based secret key plan is deficient with regards to the above fundamental focuses for the most part. By and large the content based passwords pursue the accompanying rules: 
a. At any rate 8 characters in length and alphanumeric.

b. Ought not be anything but difficult to identify with the client (for example last name, phone number, birth year).

c. Ought not be a word that can be found in a lexicon or open word reference.

d. Should consolidate upper and lower case letters and digits.

The biometric framework was presented et al.[2], As an option in contrast to the customary secret word based plan. This depends upon one of a kind highlights unaltered amid the existence time of a human, for example, fingerprints, iris and so on the mind-boggling expense of extra gadgets required for recognizable proof procedure et al.[2] is the serious issue of biometric as a validation plot. On the off chance that the gadgets are not strong the false-positive and false negative rate may likewise be high. Biometric frameworks are defenseless against replay assault (by the utilization of sticky buildup left by finger on the gadgets), which decreases the security and ease of use levels. By presenting token-based verification plans, late advancements have endeavored to defeat biometric weaknesses.

Token put together frameworks depend with respect to the utilization of a physical gadget, for example, smartcards or electronic-key for verification reason et al.[9]. The customary secret word based framework may likewise be utilized in Token based framework. Token based frameworks are helpless against man-in-the centre assaults where an interloper blocks the client's session and records the certifications by going about as an intermediary between the client and the validation gadget without the learning of the client et al.[9]. Graphical based passwords are acquainted as an option with above plans to determine security and ease of use confinements referenced in these plans.

Graphical-based secret key methods have been acquainted as a potential option with content based strategies, due to by the way that people can recollect pictures superior to content et al.[8]. Therapists additionally demonstrated that pictures are more essential than content. In this manner, graphicalbased validation plans have higher ease of use than other confirmation methods. Then again, it is likewise hard to break graphical passwords utilizing ordinary assaults, for example, word reference assault, beast power and spyware which have been influencing content based and token-based validation. In this way, there were higher sequrity in graphical based confirmation conspire than other verification plans. When all is said in done, the graphical secret phrase procedures can be characterized into two classes: acknowledgment based and review based graphical strategy et al.[1].In acknowledgment based frameworks, a gathering of pictures are shown to the client and he needs to clicked or contacted a right picture in a specific request for acknowledge verification. A few instances of acknowledgment based framework are Awase-E framework, AuthentiGraph, and Pass faces framework. Despite the fact that Awake-E framework has a higher convenience, because of the extra room required for pictures and furthermore the framework can't endure replay assault it is hard to execute. The business framework Pass faces et al.[1] uses pictures of human appearances.
Davis, et al.[3] dealt with such a plan and closed, that client's secret phrase determination is influenced by race and sexual orientation. This makes the Passfaces' secret word to some degree unsurprising. Despite the fact that an acknowledgment based graphical secret word is by all accounts simple to recollect, which expands the convenience, it isn't totally verify. It needs a few rounds of picture acknowledgment for verification to give a sensibly huge secret word space, which is repetitive et al.[3]. Likewise, clearly acknowledgment based frameworks are powerless against replay assault and mouse following as a result of the utilization of a fixed picture as a secret word. In this manner, we think about these disadvantages in our proposed framework, which beats the issues of review based plans as well.

\section{ANALYSIS OF PROBLEM}

As we probably are aware the biometric framework was presented, as an option in contrast to the content base secret phrase conspire. But since of it's mind-boggling expense, it is difficult to execute. So token based plan was presented. Token put together frameworks depend with respect to the utilization of a physical gadget, for example, smartcards or electronic-key for verification reason. Graphical-based secret key methods are acquainted as a potential option with content based strategy since we know the way that people can recall pictures superior to content. As a rule, the graphical secret phrase systems can be grouped into two classes: review based and acknowledgment based graphical procedures. In review based frameworks, the client is approached to repeat something that he/she made or chose before amid the enlistment stage. In acknowledgment based frameworks, a gathering of pictures are shown to the client and an acknowledged validation requires a right picture being clicked or contacted in a specific request, yet there are a few downsides of these frameworks, for example,

a. Alphanumeric passwords have issues, for example, client may overlooked the secret word, lexicon assault, key lumberjack, helpless against speculating, bear surfing and social building.

b. The serious issue of biometric as a confirmation plan is the staggering expense of extra gadgets required for distinguishing proof procedure.

c. Despite the fact that an acknowledgment based graphical secret word is by all accounts simple to recollect, which builds the ease of use, it isn't totally verify. It needs a few rounds of picture acknowledgment for validation to give extremely enormous secret word space, which is monotonous. In this proposed work, we exceptionally centre just around "Information based validation" kinds of confirmation.

\section{MODULE IMPLEMENTATION}

Modules

1. Login Authentication

2. New User Registration

3. Pass Points Module 
4. Signaled Click Points Module

5. Powerful Cued Click-Points

\section{Login Authentication}

Login validation is utilized to check whether the client is an approved individual to utilize the framework. For each client have been new username and secret word with one of a kind number is given through graphical framework which they can access and checking their confirmation subtleties of clients. In this undertaking can get to the client should give the right username and secret key dependent on graphical pictures. The various kinds of clients are

- Administrator

- Users

\section{New User Registration}

The enlistment login module handles standard client enrollment and login usefulness. In the enrollment stage the new understudy can enlist the subtleties and get the administration, if there is any new client they can make the new login id, in enrollment the new client must give full insights regarding the name, email, portable number, at long last they will get the client name and secret word graphically.

\section{Pass Points Module}

In this module dependent on unique thought, Pass Points $(\mathrm{PP})$ is a tick based graphical secret phrase framework where a secret phrase comprises of an arranged grouping of five snap focuses on a pixel-based picture. To sign in, a client must snap inside some framework characterized resilience locale for each snap point. The picture goes about as a prompt to enable clients to recollect their secret key snap focuses.

\section{Prompted Click Points Module}

Prompted Click Points (CCP) will create as an elective snap based graphical secret key plan where clients select one point for each picture for five pictures. The interface shows just one picture at once; the picture is supplanted by the following picture when a client chooses a tick point. The framework decides the following picture to show dependent on the client's snap point on the present picture. The following picture showed to clients depends on a deterministic capacity of the point which is presently chosen. It currently introduces a coordinated signaled review situation where each picture triggers the client's memory of the a single tick point on that picture. Furthermore, if a client enters a wrong snap point amid login, the following picture showed will likewise be mistaken. Genuine clients who see an unrecognized picture realize that they made a blunder with their past snap point. Then again, this certain criticism isn't useful to an assailant who does not know the normal succession of pictures.

\section{Convincing Cued Click-Points}

To address the issue of hotspots, Persuasive Cued Click Points (PCCP) was proposed. As with CCP, a secret phrase comprises of five snap focuses, one on every one of five pictures. Amid secret word creation, the majority of the picture is diminished aside from a little view port zone that is haphazardly situated on the picture. Clients must choose a tick point inside the view port. On the off chance that they can't or reluctant to choose a point in the present view port, they may press the Shuffle catch to arbitrarily reposition the view port. The view port aides clients to choose progressively irregular passwords that are more averse to incorporate hotspots. A client who is resolved to achieve a specific snap point may in any case mix until the view port moves to the particular area, yet this is a tedious and progressively monotonous procedure.

\section{CONCLUSION AND FUTURE WORK}

This plan shows guarantee as a usable and paramount validation system. By exploiting clients' capacity to perceive pictures and the memory trigger related with seeing another picture, CCP has focal points over PassPoints as far as convenience. Being signaled as each picture is appeared and recalling just a single tick point for every picture seems simpler than recollecting an arranged arrangement of snaps on one picture. In our little examination gathering, clients emphatically favored CCP.

We trust that CCP offers an increasingly secure option to PassPoints. CCP builds the outstanding task at hand for assailants by driving them to initially obtain picture sets for every client, and after that direct hotspot investigation on every one of these pictures. Besides, the framework's adaptability to build the general number of pictures in the framework enables us to discretionarily expand this remaining task at hand. Future work ought to incorporate a careful appraisal of the suitability of CCP as a confirmation component, including a long haul investigation of how these passwords work practically speaking and whether longer CCP passwords would be usable. The security of CCP additionally merits nearer examination, and should address how assailants may abuse the rise of hotspots.

\section{FUTURE ENHANCEMENT}

In the pictures examination found that there were not many critical contrasts among a few pictures of ordinary scenes. Utilizing direction from brain science just as instinct one might probably pick pictures that are adequately great secret key pictures and maintain a strategic distance from even from a pessimistic standpoint pictures that meddle with memorability. In any case, further work on secret key pictures is expected to decide to what degree pictures have "problem areas" that pull in numerous clients to pick secret phrase focuses in a similar little regions. In the event that problem areas happen regularly, at that point they diminish entropy of the framework. This wonder has been appeared in face acknowledgment graphical passwords, yet the threat might be less in our framework with great selection of pictures to keep away from problem areas. We intend to start concentrating problem areas by gathering countless secret key focuses on different pictures.

Blue Eyes Intelligence Engineering

\& Sciences Publication 


\section{REFERENCES}

1. Xiaoyuan, S., Z. Ying, et al. (2005). "Graphical passwords: a survey", Computer Security Applications Conference, $21^{\text {st }}$ Annual.

2. Pierce JD, Jason G. Wells, Matthew J. Warren, \& David R. Mackay. (2003). "A Conceptual Model for Graphical Authentication", 1st Australian Information security Management Conference, 24 Sept. Perth, Western Australia, paper 16.

3. Dirik, A. E., N. Memon, et al. (2007). "Modeling user choice in the Pass Points graphical password scheme", Proceedings of the 3rdsymposium on Usable privacy and security. Pittsburgh, Pennsylvania, ACM.

4. Wells, Jason; Hutchinson, Damien; and Pierce, Justin, "Enhanced Security for Preventing Man-in-the-Middle Attacks in Authentication, formation Security Management Conference.

5. Masrom, M., F. Towhidi, et al. (2009). "Pure and cued recallbased graphical user authentication", Application of Information and Communication Technologies, 2009. AICT 2009. International Conference.

6. S. Wiedenbeck, J. Waters, J.C. Birget, A. Brodskiy, N. Memon, “PassPoints: Design and longitudinal evaluation of a graphical password system", International J. of HumanComputer Studies (Special Issue on HCI Research in Privacy and Security), 63 (2005) 102-127.

7. Takada, T. and H. Koike (2003). "Awase-E: Image-Based Authentication for Mobile Phones Using User's Favorite Images", Human-Computer Interaction with Mobile Devices and Services, Springer Berlin / Heidelberg. 2795: 347-351.

8. Sadiq Almuairfi, Parakash Veeraraghavan and Naveen Chilamkurti (2011) Workshops of International Conference on Advanced Information Networking and Applications.

9. Ms.Prajakta, D.Kulkarni, Mr.C.S.Satsangi, Mr.Santhosh Easo. "Authorization using Graphical Password", IOSR Journal of Engineering (IOSRJEN) ISSN: 2250-3021 Volume 2, Issue 7(July 2012), PP 91-95.

10. Birget, J. C., H. Dawei, et al. (2006). "Graphical passwords based on robust discretization", Information Forensics and Security, IEEE Transactions on 1(3): 395-399. 